\section{Calculational subroutines for target width and movement amplitude corrections and normal distribution parameters}

\author{
JAMES L. KNIGHT, JR. \\ Purdue University, Lafayette, Indiana 47907
}

Fitts (1954) proposed an index of difficulty (ID) metric which reflects the information generated in executing a movement of specified amplitude to a target of specified width. The formula developed by analogy to the information theory of continuous signals states that ID $=\log _{2} 2 \mathrm{~A} / \mathrm{W}$, where $\mathrm{A}$ is movement amplitude and $\mathrm{W}$ is target width. The validity of this metric rests on several well known assumptions about movement performance. In particular, the spatial distribution of movement endpoints is assumed to be normal, and centered about the middle of the "target" of the movement.

Welford $(1968$, p. 147) has pointed out that the information content of a normally distributed signal is approximately $4.1333 \sigma$ (i.e., $\sigma \sqrt{2 \pi \mathrm{e}}$ ), where $\sigma$ is the standard deviation of the distribution. Therefore, if target width is equal to $4.133 \sigma$, then target width accurately reflects the information in the distribution of movement endpoints, and ID will be properly calculated. In this case, about $96 \%$ of the movements should hit the target, since \pm 2.067 o (i.e., $4.133=2 \times 2.067$ ) includes $96 \%$ of the normal distribution. Thus, if more (or less) than about $4 \%$ errors are made, nominal target width is an inaccurate reflection of the information in the movement endpoint distribution and a correction is necessary to derive an "effective" target width equal to $\pm 2.067 \sigma$ for use in the ID formula above. For example, if more than $4 \%$ misses are made, effective target width would be wider than the nominal target width intended by the experimenter.

Furthermore, a person may consistently overshoot (or undershoot) the intended target, thus producing an effective movement amplitude longer (or shorter) than the amplitude intended by the experimenter. Thus, an additional correction must be applied before computing ID.

Typically, corrections are made by consulting tables of the normal distribution function and determining a $z$ score associated with the probabilities of overshooting and undershooting the target. From these, both effective

Printouts of subroutines EFTARG and NORMAL are available upon request from James Knight, Department of Psychological Sciences, Purdue University, West Lafayette, Indiana 47907. These subroutines were developed under auspices of NIMH Grant MH-26302. target width and effective movement amplitude may be calculated. This is a laborious procedure when many trials and targets are involved. A subroutine, EFTARG, written in FORTRAN IV, automatically computes appropriate corrections. EFTARG is called with the number of on-target hits, the number of overshoots, the number of undershoots, and the measured target width (i.e., the nominal width of the target as specified by the experimenter in whatever length units are appropriate). EFTARG returns the location of the mean of the distribution of movement endpoints and an effective target width covering \pm 2.067 of this distribution. The location of the distribution mean is specified as a distance to the nominal target center in the same units used to specify intended target width. If the distance is shifted toward the "overshoot" side of the target, this distance is positive; if it is on the "undershoot" side, this distance is negative. An error condition results if there are no overshoots or undershoots (since corresponding $z$ scores would be plus or minus infinity). If this happens, the subroutine returns $-1 \times$ nominal target width and distribution mean location $=0$ (i.e., centered on target midpoint).

The main subroutine, EFTARG, makes use of a second FORTRAN IV subroutine of general utility in other applications. This subroutine, NORMAL, calculates parameters of a normal distribution. It functions in two modes: (1) The user can pass a $\mathrm{z}$ score to the subroutine and NORMAL returns with the area under the normal density function to the left of the $\mathrm{z}$ score (i.e., the integral from $-\infty$ to the $z$ score); (2) the user can pass an area under the density function (i.e., a probability), and the subroutine will return a $z$ score such that the area to the left of the $\mathrm{z}$ score is the given probability. In both modes, the subroutine will also return the ordinate of the density function at the $\mathrm{z}$ score specified (or calculated in Mode 2).

To use this subroutine the user simply passes either a $\mathrm{z}$ score or a probability and the subroutine returns with the complementary value. This subroutine uses approximation techniques to derive its answers and the results are usually accurate to about five decimal places except beyond +6 and -6 standard deviations, where probabilities to the left of the $\mathrm{z}$ score are fixed at 1 and 0 , respectively.

\section{REFERENCES}

FrrTs, P. M. The information capacity of the human motor system in controlling the amplitude of movement. Journal of Experimental Psychology, 1954, 47, 381-391.

WELFORD, A. T. Fundamentals of skill. London: Methuen, 1968. 
SUBROUTINE EFTARG (HITS, OVERS, UNDERS, SIZE, CENTER)

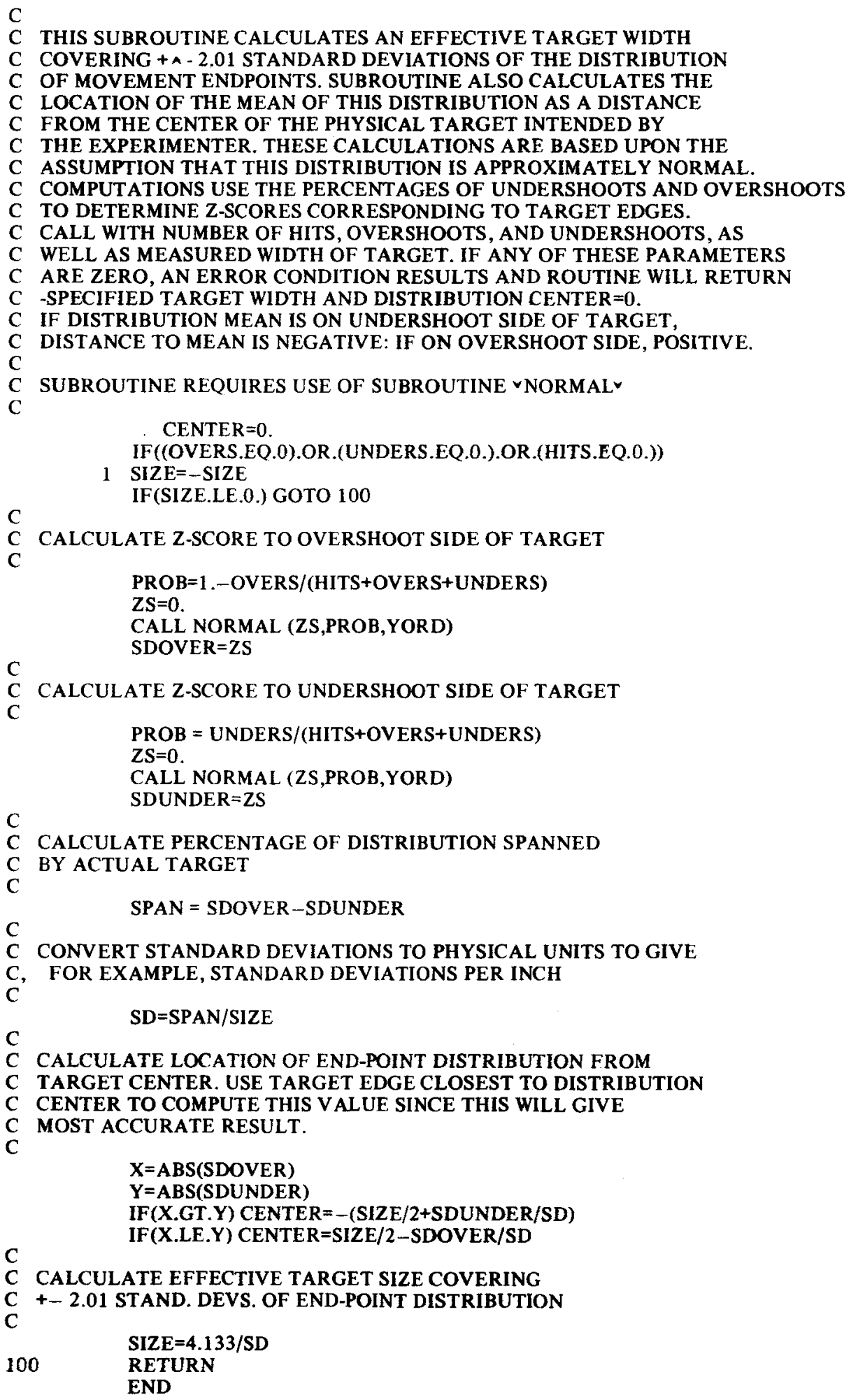


SUBROUTINE NORMAL (ZS,PROB,YORD)

C ROUTINE TO COMPUTE PARAMETERS OF NORMAL DISTRIBUTION

C CALL WITH Z-SCORE (ZS) OR PROBABILITY TO LEFT OF A

C UNKNOWN Z-SCORE (PROB). OTHER PARAMETER SHOULD BE ZERO

C ROUTINE RETURNS COMPLIMENTARY VALUE AND ALSO ORDINANT

C OF NORMAL DISTRIBUTION AT SPECIFIED (OR CALCULATED) Z-SCORE.

C FOR Z-SCORES LESS THAN -6 ROUTINE RETURNS 0 FOR PROBABILITY

C FOR Z-SCORES GREATER THAN 6, ROUTINE RETURNS 1 FOR PROBABILITY.

C

C

ILOOP $=10$

IF (PROB.EQ.0.) ILOOP=1

DELT $=-1$

$\mathrm{X}=0$.

DO $100 \mathrm{I}=1$,ILOOP

$\mathrm{DELT}=-.1 * \mathrm{DELT}$

110

$\mathrm{X}=\mathrm{X}+\mathrm{DELT}$

IF (PROB.EQ.0.) $X=Z S$

$Z=0$

IF(X.EQ.0.) GO TO 2

$Z=1$.

$\mathrm{Y}=\mathrm{ABS}(\mathrm{X}) * .5$

IF(Y.GE.3.) GOTO 2

IF(Y.GE.1.) GOTO 1

$\mathrm{W}=\mathrm{Y}^{*} \mathrm{Y}$

$Z=r((()((0.000124818987 * W$

$1-.001075204047) * W+0.005198775019) * W$

$2-.019198292004) * W+0.059054035642) * W$

$3-.151968751364) * W+0.319152932694) * W$

$4-.531923007300) * \mathrm{~W}+0.797884560593) * \mathrm{Y} * 2$.

GOTO 2

1

$$
\mathrm{Y}=\mathrm{Y}-2 \text {. }
$$

$\mathrm{Z}=((()(()((c(()-.000045255659 * \mathrm{Y}$

$\left.1+.000152529290)^{*} \mathrm{Y}-0.000019538132\right) * \mathrm{Y}$

$2-.000676904986) * Y+0.001390604284) * Y$

$3-.000794620820) * Y-0.002034254874) * Y$

$4+.006549791214) * Y-0.010557625006) * Y$

$5+.011630447319) * Y-0.009279453341) * Y$

$6+.005353579108) * Y-0.002141268741) * Y$

$7+.000535310849) * Y+0.999936657524$

$\mathrm{PN}=(1.0-\operatorname{SIGN}(Z, X))^{*} .5$

$P Q=.5-P N$

IF(PROB.EQ.0.) GOTO 100

IF(DELT.LT.0.) GOTO 101

IF(PN.GT.PROB) GOTO 110

GOTO 100

101 IF (PN.LT.PROB) GOTO 110

100 CONTINUE

IF(PROB.EQ.0) $\mathrm{PROB}=1 .-\mathrm{PN}$

IF(ZS.EQ.0) ZS $=-X$

$\mathrm{X}=-5 *(\mathrm{ZS} * * 2)$

$X 1=2 * 3.1415927$

YORD $=E X P(X) / S Q R T(X 1)$

120

RETURN

END

CKG0646 14.45.56.LQ23 2 PAGES 